\title{
A Call for Guidance in the Use of Left Ventricular Assist Devices in Older Adults
}

\author{
Caroline A. Vitale, MD, ${ }^{*}$ Rashmi Chandekar, MD, ${ }^{*}$ Phillip E. Rodgers, $M D,{ }^{\S}$ \\ Francis D. Pagani, MD, PhD," and Preeti N. Malani, MD, MSJ"
}

[See Editorial Comments by Dan D. Matlock, MD, MPH pp 154-155]

\begin{abstract}
Left ventricular assist devices (LVADs) are approved as "destination therapy" (permanent use without plans for transplantation) in individuals with advanced heart failure who are not candidates for a cardiac transplant; as such, these devices are increasingly being used in older adults. Although LVADs have been shown to increase quality of life and survival, the associated treatment burdens and complications deserve careful consideration. The current study illustrates myriad clinical challenges that can arise during long-term mechanical support using an older adult case history. Current data on LVAD use in older adults is reviewed, and a discussion of relevant points to consider before LVAD implantation in older adults, including advance care planning, assessment of gait and cognition, and the potential for substantial caregiver burden, is undertaken. J Am Geriatr Soc 60:145-150, 2012.
\end{abstract}

Key words: left ventricular assist devices; quality of life; older adults; destination therapy

From the "Division of Geriatric Medicine, University of Michigan Health System, ${ }^{\dagger}$ Geriatric Research, Education and Clinical Center, Veterans Affairs Ann Arbor Healthcare System, Ann Arbor, Michigan; ${ }^{\star}$ Division of General Internal Medicine, Department of Internal Medicine, Virginia Commonwealth University Health System, Richmond, Virginia;

${ }^{\S}$ Department of Family Medicine, University of Michigan Health System, "Section of Cardiac Surgery, Department of Surgery, University of

Michigan Health System, and "Division Infectious Diseases, Department of Internal Medicine, University of Michigan Health System, Ann Arbor, Michigan.

Address correspondence to Caroline A. Vitale, Department of Internal Medicine, Division of Geriatric Medicine, University of Michigan Health System, Geriatric Research, Education and Clinical Center, Veterans Affairs Ann Arbor Healthcare System, 2215 Fuller Road, GRECC (11G), Ann Arbor, MI 48105. E-mail: cavitale@umich.edu

DOI: $10.1111 / \mathrm{j} .1532-5415.2011 .03740 . \mathrm{x}$
$\mathrm{O}$ riginally indicated as a bridge to transplantation, left ventricular assist devices (LVADs) have become more common as elective, permanent "destination therapy" (DT) for individuals with advanced heart failure who are not eligible for cardiac transplantation. "Destination therapy" means permanent implantation of an LVAD without subsequent plans for cardiac transplantation. In this regard, DT can be thought of as a definitive therapy (as opposed to the use of an LVAD as a temporary therapy before heart transplant) that is potentially life sustaining or life prolonging for individuals with advanced heart failure. Recent studies suggest positive effects of DT on symptom management, quality of life, and functional capacity, prompting consideration of mechanical circulatory support as a viable treatment option for individuals with advanced heart failure seeking palliation. Given the high prevalence of advanced heart failure in older adults, ${ }^{1}$ coupled with dramatic advances in LVAD technology, it is not surprising that the mean age of individuals undergoing LVAD placement has also increased. ${ }^{2}$ For these reasons, clear guidance regarding the use DT in older adults that addresses risks, benefits, and burdens is imperative.

The current article presents an instructive case that prompts discussion of recipient selection for LVAD placement in an effort to better identify older adults who are likely to benefit from this therapy and to recognize those with a low likelihood of survival or improvement in quality of life. A review of LVAD basics, along with potential benefits, risks, and treatment burdens, in older adults is undertaken, keeping in mind that an important challenge to understanding current practices is the dynamic nature of this rapidly evolving technology.

\section{CASE SUMMARY}

Mr. J was a 70-year-old man who presented with a past medical history of a previous stroke and residual right-sided 
weakness, a history of two mechanical falls in the past year, extensive coronary artery disease resulting in advanced congestive heart failure refractory to maximal medical management, and associated poor endurance. After comprehensive evaluation, he underwent placement of a durable, implantable LVAD on January 21, 2009, intended as definitive therapy for his advanced heart failure. After an extended hospital course complicated by postoperative delirium, he was discharged to subacute rehabilitation on Postoperative Day 41. Although his cognition improved during rehabilitation, he continued to require substantial assistance with activities of daily living upon discharge home from subacute rehabilitation because of deconditioning and gait instability. With close follow-up with the LVAD team, Mr. J was able to live at home for approximately 10 months. Although dependent on his wife for many of his care needs, especially the care related to his LVAD, he gained symptomatic improvement in his dyspnea and fatigue.

Approximately 10 months after LVAD implantation, he attempted to rise from a seated position, tripped, and fell, dislodging his LVAD driveline in the process. $\mathrm{He}$ called out to his wife, who found him unresponsive on the floor. She retrieved the LVAD backup controller and connected it. He was disconnected from pump for 6 minutes. Several minutes later, emergency medical services arrived and initiated cardiopulmonary resuscitation. He was transported to a local hospital and was later transferred to the original implanting center in an air ambulance, where he stabilized.

This episode left him with significant anoxic brain injury. He was minimally responsive for several days, followed by some improvement in mentation, but episodes of confusion persisted. He also exhibited combative behavior requiring behavioral and pharmacological management. His family, although devastated, remained hopeful for even a modest recovery so that he might be able to return home. He was discharged to subacute rehabilitation, where he required close supervision because of residual cognitive impairment from the anoxic episode. Despite best efforts, there were several inconsistencies in nursing care in the subacute facility because of lack of familiarity with LVADs that resulted in rehospitalization.

During the next year, Mr. J lived at home, with his wife as his primary caregiver, providing 24-hour supervision and personal care, with intermittent help from their adult children. He remained severely cognitively and physically impaired, with complete dependence in all activities of daily living. His care needs and medical expenses resulted in considerable financial burden for his family. His wife had stopped working to care for him, and their finances were such that they were unable to hire any outside help. During this year, he was hospitalized seven times. Because of his continued overall decline, repeated hospitalizations, and substantial caregiver stress on the part of his wife, the geriatric medicine and palliative care teams were consulted and provided input on several occasions. His wife and sons were initially reluctant to entertain the idea of hospice because of deeply held cultural beliefs, but as he continued to decline, his family eventually accepted hospice services, and he died at home 2 weeks later.

\section{DISCUSSION}

\section{Overview}

An LVAD, the most common type of durable mechanical circulatory assist device, is a mechanical pump that provides support to the left ventricle to maintain adequate blood flow in individuals with advanced heart failure. LVADs require surgical implantation through a median sternotomy and consist of an internal pump with a percutaneous lead connecting to an external controller. The external controller requires constant connection to a power source (batteries or electrical line power source). LVADs generally fall into two types: pulsatile flow and continuous flow. The first-generation pulsatile-flow devices were designed to mimic human hemodynamics by generating pulsatile circulation. These devices are larger, with multiple moving parts that can wear out, and have been associated with higher rates of complications and higher mortality than newer devices. The smaller, quieter, second-generation devices using continuous-flow technology provide more durability because they have fewer moving parts, allow for implantation into recipients with a smaller body size, and have resulted in longer survival than the first-generation devices. ${ }^{2,3}$

Individuals being considered for LVAD implantation are considered to fall into one of several categories. The two main categories include bridge to transplant (BTT) and destination therapy (DT). Another category, called "bridge to candidacy" is designated for those receiving LVADs in whom a transplant might be considered in the future, depending on stability after the LVAD placement. ${ }^{2}$ The decision regarding DT versus BTT can be a dynamic process, with recipients moving between groups. For instance, some recipients feel so well after LVAD placement that they decline transplant. Others begin as transplant candidates but are no longer felt to be appropriate after device placement.

Before Food and Drug Administration approval of LVADs for DT, many older adults, including Mr. J, would not have been considered for LVAD implantation because the approved indication was primarily BTT. Although there is not a firm age limit for heart transplantation, there is an effective limit based on the overall clinical picture, and rarely would someone in their 70s be considered for transplant. In a sense, the expansion of LVAD indications to include DT has effectively removed this built-in age deterrent, opening the door to implantation in much older (and frailer) individuals with advanced heart failure. Current data from the Interagency Registry for Mechanically Assisted Circulatory Support (INTERMACS) registry, an ongoing National Heart Lung and Blood Institutesponsored collaborative database of LVAD implantations in the United States, ${ }^{2}$ indicates that there has been a dramatic increase in the number of people receiving DT in 2010 from previous years. ${ }^{2}$ The mean age of individuals undergoing DT from June 2006 to January 2010 was 61.7 (range 23-82), compared with 52.7 (range 19-88) for individuals receiving LVADs for all other indications. ${ }^{2}$ With rapidly evolving technology, and with DT becoming available in many centers (69 centers across the United States have been designated DT centers by CMS), ${ }^{2}$ a continued 
increase in the numbers of older adults considered for this therapy can be expected.

\section{Outcomes}

With optimal medical management, individuals with advanced heart failure undergoing chronic inotropic therapy have a reported 6-month baseline mortality of almost $50 \%{ }^{4}$ Of individuals who are not deemed eligible for cardiac transplantation, DT has been shown to extend life and to improve certain quality-of-life (QOL) measures. These outcomes appear to be improving, particularly in individuals receiving the newer continuous-flow LVADs, as demonstrated in the HeartMate II (Thoratec Corp., Pleasanton, CA) destination therapy trial ${ }^{5}$ and the most recent INTERMACS registry data. ${ }^{2}$ Although the 1- and 2-year survival rates for participants in the HeartMate II trial (comparing continuous-flow devices with previously approved pulsatile devices in individuals ineligible for heart transplant) were $68 \%$ and $58 \%$, respectively, ${ }^{5}$ further experience with newer continuous-flow devices has resulted in a 1-year survival rate of $74 \% .^{2}$ This improvement in survival is thought in part to reflect recipient selection, with current trends moving toward implanting LVADs into less-sick individuals, somewhat earlier in the trajectory of advanced heart failure. ${ }^{6}$ In addition, age alone has recently been identified as an important factor in predicting clinical outcomes. Older age (70 vs 60$)$ has shown to be an independent predictor of death throughout the duration of LVAD therapy. ${ }^{2}$ Conversely, younger age is emerging as an important predictor of who will thrive clinically over the long term with LVAD support. ${ }^{7}$

With better recipient selection, it has been proposed that individuals with advanced heart failure might benefit from prolonged survival and enhanced QOL with LVAD therapy, despite its burdensome nature. ${ }^{8}$ Substantial improvements in QOL have been reported, especially with the newer continuous-flow devices, ${ }^{9}$ although these QOL outcomes for older DT recipients have not been analyzed separately. Despite this, clinically meaningful improvements in heart failure-related QOL measures using the Minnesota Living with Heart Failure Questionnaire ${ }^{10}$ and the Kansas City Cardiomyopathy Questionnaire ${ }^{11}$ were seen in DT recipients in the HeartMate II trial. ${ }^{8,9}$ Improvements in functional status measures were also observed, as measured using New York Heart Association (NYHA) functional class, metabolic equivalents (METs), and 6-minute walk test.' Whereas most participants had NYHA Class IV symptoms at baseline, $80 \%$ of surviving DT recipients improved to NYHA Class I or II 6 months after LVAD implantation. Although substantial improvement in 6-minute hall walk test was also reported, only $34 \%$ of participants were able to perform the test at baseline. ${ }^{9}$

In light of these improvements in survival and heart failure-related QOL parameters, it is important to examine the nature and frequency of durable mechanical support device complications and ongoing treatment burdens as they relate to older adults. As illustrated in part by the current case, relevant outcomes in older adults also include QOL as related to treatment burden and complications as well as psychological and cognitive status. Caregiver burden, place
Table 1. Complications Observed in Individuals with Continuous-Flow Left Ventricular Assist Devices (LVADs) in the Heartmate II Destination Therapy Trial at 24 Months $(\mathrm{N}=133)^{5}$

\begin{tabular}{lc}
\hline \multicolumn{1}{c}{ Subgroup } & n (\%) \\
\hline LVAD-related infection & $47(35)$ \\
Sepsis & $48(36)$ \\
Bleeding requiring surgery & $40(30)$ \\
Stroke & $24(18)$ \\
Ischemic & $11(8)$ \\
Hemorrhagic & $15(11)$ \\
Other neurological event & ${ }^{*}$ \\
Rehospitalization $^{\dagger}$ & $29(22)$ \\
Pump replacement $^{\dagger}$ & $107(94)$ \\
\hline
\end{tabular}

"Transient ischemic attacks and events other than stroke.

${ }^{\dagger}$ Due to percutaneous lead damage.

of care, and overall financial burden to individuals, families, and society are also important considerations.

Perioperative and long-term complications of DT therapy are common (Table 1). Stroke, infection, bleeding, and device malfunction or failure requiring reoperation are recognized complications and substantial sources of morbidity and mortality, especially early after device implantation. ${ }^{5}$ Stroke and infection appear to be particularly common and can be devastating. Hypercoagulability, along with intermittent low-flow states can increase the risk of embolic stroke, transient ischemic attacks, and hypoxia with associated delirium. Although advanced heart failure alone is thought to contribute to cognitive deficits, ${ }^{12}$ impairments in neurocognitive function, including delirium, have been noted in approximately one-quarter to one-third of individuals after LVAD implantation in small observational studies. ${ }^{13,14}$ With the newer continuous-flow devices, early observations are consistent with trends toward improvements in executive functioning and memory 1 and 6 months after LVAD implantation without decline in other domains on neuropsychiatric testing. ${ }^{15}$ Although the possibility of a lower incidence of delirium and other neurocognitive deficits with newer devices may be anticipated, these results need to be interpreted with caution because they are not reported specifically in elderly adults receiving LVADs.

Another important consideration in older adults is the reported greater incidence of bleeding (gastrointestinal bleeding in particular) observed more recently with continuous-flow LVADs. ${ }^{6}$ Individuals with continuous-flow devices universally require long-term anticoagulation, which may contribute to greater bleeding risk postoperatively and after discharge.

Other potential treatment burdens include the special attention needed for the care of percutaneous drivelines and the need for continued external power supply. As the current case illustrates, attention to protecting the driveline from trauma must be paid at all times to avoid disconnection or mechanical malfunction. Living with an LVAD can result in some restrictions in position and decreased mobility due to the presence of the driveline and external power supply. Not surprisingly, the day-to-day care and attention that the percutaneous driveline demands can be 
problematic for frail older adults, who are assumed to have low functional and cognitive reserve.

Adherence to a complex treatment regimen is compulsory and also potentially burdensome. This includes the need to understand and respond to LVAD system signals, adhere to infection precautions, and maintain close followup with heart failure specialists in LVAD centers that may be distant from home. In contrast to individuals receiving an LVAD as a BTT, individuals and their caregivers take on this substantial change in lifestyle as a permanent change for the duration of the individual's life. Not surprisingly, adjustment disorder and major depression have been noted to occur with greater prevalence after LVAD implantation. ${ }^{13,14,16}$

\section{Participant Selection and Considerations in Older Adults}

For any individual being considered for potential LVAD therapy, current standards call for an extensive, multidisciplinary, preoperative assessment by a team that includes, at a minimum, a heart failure physician, a dietitian, and a pharmacist. ${ }^{3}$ Careful assessment of an individual's medical status is undertaken for optimal recipient selection and timing of LVAD implantation in an effort to minimize perioperative risk. Initially, the nature of the individual's heart failure status is risk stratified using a scoring system based on outcomes from the INTERMACS registry. ${ }^{2}$ The mostrobust data support consideration for LVAD placement in individuals who are stable on inotropic support and not those who have cardiogenic shock or other high-risk status, including major end-organ failure, prolonged mechanical ventilation, sepsis, and hemodynamic instability. ${ }^{3}$

Once an individual is determined to be a potential LVAD candidate based on heart failure status, further assessment of perioperative risks and potential longer-term treatment benefits and burdens is undertaken. In addition to preoperative assessment of multiple parameters, including hemodynamics, hepatic and renal function, hematological and coagulopathic measures, and nutritional status, it is suggested that the individual's social network, including adequacy of caregiver support, housing, transportation, and overall adherence to treatment, be assessed and factored into any decision regarding candidacy. ${ }^{3}$ Current standards also call for the assessment of the presence of psychiatric disorders, history of substance abuse, or any neurological condition that might impair the individual's ability to care for the external LVAD system, ${ }^{3}$ although specific assessment protocols and tools used for neurological, functional, psychiatric, and psychosocial assessments are not standardized across centers.

The current case highlights several points worth exploring as they relate to older adults. At baseline, the individual described above had evidence of gait impairment due to a previous stroke, sustaining two mechanical falls in the year before LVAD implantation. Currently accepted Centers for Medicare and Medicaid Services DT criteria $^{17}$ are based on cardiopulmonary physiological parameters (Table 2). Neither these criteria nor current expert consensus on recipient selection for $\mathrm{DT}^{3}$ formally address whether the presence of certain geriatric syndromes, such as gait impairment, might preclude LVAD eligibility. Although falls from multiple causes are common in older adults, ${ }^{18}$ older adults with heart failure have a greater prevalence of geriatric conditions including gait impairment and are more likely to sustain an injury when falling. ${ }^{19}$ In retrospect, perhaps this individual's preexisting gait impairment and history of falls could have been considered a contraindication to LVAD implantation, given his subsequent fall resulting in driveline disconnection and anoxic encephalopathy. Although he underwent a full physical therapy evaluation as part of his pre-LVAD assessment, his gait impairment (hemiparesis necessitating a cane) was deemed to be mild and did not affect his candidacy for DT. It might be argued that improvements in exercise tolerance and ambulation, as evidenced by documented improvements in the 6-minute hall walk test noted in the HeartMate II DT trial, may be reasonable expectations in individuals who are ambulatory before receiving LVAD destination support. ${ }^{9}$

As with most geriatric syndromes and conditions, the role of impaired gait and balance is underexplored but remains an important area for investigation as greater numbers of older adults are offered LVAD support. Slow gait speed has recently been found to be a useful predictor of mortality and major morbidity in older adults undergoing cardiac surgery. ${ }^{20}$ This simple measure, defined as time taken to walk $5 \mathrm{~m}$ for longer than 6 seconds, has been found to be a useful global measure to detect frailty in individuals with multiple comorbidities and deserves further study as a potential useful predictive tool for older adults being considered for DT.

As illustrated with Mr. J's case, primary caregivers and family members of individuals with DT can experience prolonged caregiver stress, with the need for vigilant monitoring, especially in individuals who have sustained a near-catastrophic complication such as $\mathrm{Mr}$. J or other common complications such as stroke or infection. Little is known about the experience of caregivers caring for individuals with DT, in which intensive caregiving needs can be prolonged. One qualitative study characterizing the experience of caregivers for people with BTT describes recurrent themes best characterized as "commitment," indicating that they would do it all again; "sacrifice," in which they describe social isolation, loss of employment, and financial strain; and "moving beyond," in which they are able to adapt and accept their intensive caregiving roles. ${ }^{21}$ These experiences and themes may not be fully applicable to caregivers of DT recipients, because the BTT is usually viewed as a situation to be endured temporarily.

Evidence of overall cost-effectiveness of mechanical support is uncertain at best. The rapid progress in LVAD technology over the last few years, characterized by documented improvements in survival and heart failure-related QOL measures, limit current assessments of cost-effectiveness. A 2008 study analyzing outcomes and costs of LVADs in Medicare beneficiaries concluded that LVADs were associated with substantial early mortality, morbidity, and high costs. ${ }^{22}$ That the newer continuous-flow devices were not included in this study appears to be a major limitation. Further analysis is needed given the recent dramatic shift to the almost-exclusive use of the newest-generation devices that most centers are implanting, 
although as Medicare spending associated with procedures and device implantation comes under greater political and public scrutiny, ${ }^{23}$ the high societal costs associated with LVAD support also need to be carefully weighed.

An important aspect of the pre-LVAD evaluation includes cognitive assessment to ensure adequate decisionmaking and a true informed consent process. ${ }^{24}$ Some propose full neurocognitive testing to detect impairments in executive function that can affect complex treatment needs and ability to perform instrumental activities of daily living. ${ }^{25}$ True informed consent includes discussing benefits and treatment burdens of all options (Table 3). ${ }^{24,26}$ General consensus dictates that the informed consent process take place directly with the individual and not a surrogate decision-maker, because LVADs are not curative and less-burdensome therapies exist. ${ }^{24}$

Greater use of DT in older adults provides important opportunities for greater partnership between colleagues in geriatric medicine and palliative care, with specialized heart failure teams providing longitudinal care for individuals receiving mechanical support. Such opportunities for collaboration exist during pre-LVAD evaluation and during support, when complications and comorbidities are likely to affect an individual's QOL. Formal calls for greater participation of palliative care and geriatric medicine teams in the care of individuals receiving destination mechanical support have been made. ${ }^{24,26,27}$

Table 2. Centers for Medicare and Medicaid Services Criteria for Left Ventricular Assist Device (LVAD) Destination Therapy ${ }^{16}$

New York Heart Association Class IV for $\geq 90$ days

Life expectancy $<2$ years

Not a candidate for transplantation

Symptoms have not responded to optimal medical management

for $>60$ days

Left ventricular ejection fraction $<25 \%$

Peak oxygen consumption of $<12 \mathrm{~mL} / \mathrm{kg}$ per minute or need

for continuous intravenous inotropes

Appropriate body size $\left(\geq 1.5 \mathrm{~m}^{2}\right)$ to support the LVAD implantation
Although a full discussion of the dying process of individuals receiving LVAD therapy is beyond the scope of this article, it is noted that a thoughtful dialogue about advance care planning should begin before LVAD implantation. ${ }^{26,27}$ The pre-LVAD evaluation must include a detailed discussion of individual goals and values, specifically anticipating the situation in which LVAD therapy no longer supports a level of function or QOL that is acceptable to the individual. Although it is not possible to predict exactly how this situation may arrive, most individuals know what core life functions are most important to them, for example, continuing to contribute to work, family, or social roles; being able to live at home or at least outside the hospital; or simply being able to interact with loved ones and appreciate even passive participation in meaningful activities. Goals can change over time as an individual adapts to changing capacity, so this discussion should be revisited regularly. Eliciting such information before LVAD placement is not meant to predetermine specific treatment decisions but rather to establish a framework to ensure that ongoing treatment plans are helping an individual live the way he or she wants to live.

At the University of Michigan Hospital, all recipients undergo detailed pre-LVAD evaluation by a multidisciplinary team in a process that is identical to the assessment before heart transplantation. Goals of therapy are discussed extensively, as is the need for a 24 hour companion. Advance care planning, including discussions about assigning a durable power of attorney for healthcare decision-making, is reviewed with every individual. In addition to establishing traditional advance directives, efforts to assist individuals and their surrogates with "in the moment" decision-making have been proposed and appear especially useful for LVAD-related decision-making. ${ }^{28}$ Additional steps may also assist with difficult decisions; with early involvement of palliative care before implantation, some programs encourage an LVAD candidate to consider a "preparedness plan" or palliative care plan when treatment burdens of ongoing support outweigh its benefits. ${ }^{26,29}$ In this way, the groundwork is laid for when complications might arise. Ongoing palliative care involvement can help

\section{Table 3. Elements of Informed Decision-Making in Discussions About Left Ventricular Assist Device (LVAD) Des- tination Therapy (DT): Questions to Ensure That Individuals with Advanced Heart Failure Are Making Informed Decisions ${ }^{3,11}$}

Have all treatment options been discussed, including continued optimal medical management (often with inotropic support), DT,
or palliative care?
What is the individual's understanding of his or her disease?
What are the individual's goals of care? Survival? Improved quality of life? What are the individual's hopes?
Does the individual have a clear understanding of the common complications of DT, including infection, stroke, bleeding, need
for anticoagulation, and device malfunction, and what the individual will typically experience?
Does the individual have advance directives in place, including a designated durable power of attorney for health care and a concise plan
of individual preferences for anticipated device-related complications?
Does the individual have an accurate understanding of day-to-day life with an LVAD? Has he or she talked with an LVAD recipient?
Can the individual articulate agreed-upon guidelines for deactivating his or her LVAD if treatment burdens outweigh benefits or if the
situation appears futile? Has the role of palliative care and symptom management near the end of life been discussed?
Have criteria for device change-out been discussed with the individual, including a discussion of when this would not be offered?
Have the individual and caregiver(s) received appropriate counseling regarding the substantial role of the caregiver; the need for a
back-up caregiver to be available; the responsibilities of the caregiver pertaining to the need to manage the device monitors, alarms,
and backup controls; and risk of significant caregiver burden?
Do the individual and caregiver understand the nature of the potential long-term financial burden often associated with LVAD destination therapy?


with symptom management and with transitioning to device deactivation to allow natural death in some cases. ${ }^{26}$

Mr. J's case highlights the complex nuances of DT for an older adult who was able to derive symptomatic benefit from DT for a number of months but then sustained treatment complications that included markedly poorer cognition and impaired physical function. It raises additional ethical questions about continuing LVAD therapy despite substantial treatment burden and the possibility of futility. Finally, the case underscores the substantial caregiver burden and financial strain that can befall those caring for individuals dependent on technologic therapies such as LVADs. As such interventions become more commonplace, these challenges will persist. Routine assessment of gait, functional status and cognition, degree of caregiver support, advance care planning, and early involvement of palliative care should be conducted in all older adults being considered for DT.

\section{ACKNOWLEDGMENTS}

This work was presented in poster format at the American Geriatrics Society Annual Scientific Meeting, Orlando, Florida, May 2010.

Conflict of Interest: Dr. Pagani is supported by the following grants: Principal Investigator-National Heart, Lung and Blood Institute (NHLBI) Contract: Randomized Comparison of Ventricular Assist Devices Intervention Before Inotrope Therapy; Co-investigator-NHLBI Contract: INTERMACS Registry; National Principal Investigator-ENDURANCE Trial; Randomized Comparison of HeartWare HVAD to Thoratec HeartMate II for Destination Therapy; Principal Site Investigator-ADVANCE Trial: Clinical Evaluation of the HeartWare HVAD for Bridge to Transplant Therapy. This work was supported in part by the Veterans Affairs Ann Arbor Healthcare System, Geriatric Research Education and Clinical Center, and the John A. Hartford Foundation's Center of Excellence.

Author Contributions: Vitale: Literature review, manuscript drafting, preparation, and editing. Chandekar: Drafting case report, literature review, manuscript preparation. Rodgers: Provided input regarding the structure of the case report and editing the manuscript. Pagani: Manuscript revising and review. Malani: Drafting and editing of manuscript.

Sponsor's Role: None.

\section{REFERENCES}

1. Roger VL, Go AS, Lloyd-Jones D et al. Heart disease and stroke statistics 2011 update: A report from the American Medical Association. Circulation 2011;123:e18-e209.

2. Kirklin JK, Naftel DC, Kormos RL et al. Third INTERMACS annual report: The evolution of destination therapy in the United States. J Heart Lung Transplant 2011;30:115-123.

3. Slaughter MS, Pagani FD, Rogers JG et al. Clinical management of continuous-flow left ventricular assist devices in advanced heart failure. J Heart Lung Transplant 2010;29:S1-S39.

4. Gorodeski EZ, Chu EC, Reese JR et al. Prognosis on chronic dobutamine or milrinone infusions for stage D heart failure. Circ Heart Fail 2009;2:320 -324 .
5. Slaughter MS, Rogers JG, Milano CA et al. Advanced heart failure treated with continuous-flow left ventricular assist device. $N$ Engl J Med 2009;361:2241-2251.

6. Slaughter MS, Meyer AL, Birks EJ. Destination therapy with left ventricular assist devices: Patient selection and outcomes. Curr Opin Cardiol 2011;26:232-236.

7. Baumwol J, Macdonald PS, Keogh AM et al. Right heart failure and "failure to thrive" after left ventricular assist device: Clinical predictors and outcomes. J Heart Lung Transplant 2011;30:888-889.

8. Starling RC. Improved quantity and quality of life: A winning combination to treat advanced heart failure. J Am Coll Cardiol 2010;55: 1835-1836.

9. Rogers JG, Aaronson KD, Boyle AJ et al. Continuous flow left ventricular assist device improves functional capacity and quality of life of advanced heart failure patients. J Am Coll Cardiol 2010;55:1826-1834.

10. Rector TS, Kubo SH, Cohn JN. Validity of the Minnesota Living with Heart Failure questionnaire as a measure of therapeutic response to enalapril or placebo. Am J Cardiol 1993;71:1106-1107.

11. Spertus J, Peterson E, Conard MW et al. Monitoring clinical changes in patients with heart failure: A comparison of methods. Am Heart J 2005;150:707-715.

12. Almeida OP, Flicker L. The mind of a failing heart: A systematic review of the association between congestive heart failure and cognitive functioning. Intern Med J 2001;31:290-295.

13. Shapiro PA, Levin HR, Oz MC. Left ventricular assist devices: Psychological burden and implications for heart transplant programs. Gen Hosp Psychiatry 1996;18:30S-35S.

14. Baba A, Hirata G, Yokoyama F et al. Psychiatric problems of heart transplant candidates with left ventricular assist devices. J Artific Organs 2006;9:203-208

15. Petrucci RJ, Wright S, Naka $\mathrm{Y}$ et al. Neurocognitive assessments in advanced heart failure patients receiving continuous-flow left ventricular assist devices. J Heart Lung Transplant 2009;28:542-549.

16. Eshelman AK, Mason S, Nemeh $\mathrm{H}$ et al. LVAD destination therapy: Applying what we know about psychiatric evaluation and management from cardiac failure and transplant. Heart Fail Rev 2009;14:21-28.

17. Centers for Medicare and Medicaid Services (CMS) Pub 100-03 Medicare National Coverage Determinations 2007. Available at: https://www.cms. gov/transmittals/downloads/R68NCD.pdf Accessed May 30, 2011.

18. AGS Clinical Practice Guideline. Prevention of Falls in Older Persons 2010. American Geriatrics Society [on-line]. Available at: http://www.american geriatrics.org/health_care_professionals/clinical_practice/clinical_guidelines_ recommendations/2010/ Accessed May 30, 2011.

19. Gure TR, Kabeto MU, Blaum CS et al. Degree of disability and patterns of caregiving among older Americans with congestive heart failure. J Gen Intern Med 2008;23:70-76.

20. Afilalo J, Eisenberg MJ, Morin J et al. Gait speed as an incremental predictor of mortality and major morbidity in elderly patients undergoing cardiac surgery. J Am Coll Cardiol 2010;56:1668-1676.

21. Baker K, Flattery M, Salyer J et al. Caregiving for patients requiring left ventricular assistance device support. Heart Lung 2010;39:196-200.

22. Hernandez AF, Shea AM, Milano CA et al. Long-term outcomes and costs of ventricular assist devices among Medicare beneficiaries. JAMA 2008;300:2398-2406

23. Redberg RF. Squandering Medicare's Money. Op-Ed, New York Times, May 25, 2011 [on-line]. Available at: http://www.nytimes.com/2011/05/26/ opinion/26redberg.html?_r=1 Accessed May 30, 2011.

24. Dudzinski DM. Ethics guidelines for destination therapy. Ann Thorac Surg 2006;81:1185-1188.

25. Marcus P. Left ventricular assist devices: Psychosocial challenges in the elderly. Ann Thoracic Surg 2009;88:48-49.

26. Rizzieri AG, Verheijde JL, Rady MY et al. Ethical challenges with the left ventricular assist device as a destination therapy. Philos Ethics Humanit Med 2008;3:20.

27. Swetz KM, Freeman MR, Mueller PS et al. Clinical management of continuous-flow left ventricular assist devices in advanced heart failure. J Heart Lung Transplant 2010;29:1081. Comment on J Heart Lung Transplant. 2010;29:S1-39.

28. Sudore RL, Fried TR. Redefining the "planning" in advance care planning: Preparing for end-of-life decision making. Ann Intern Med 2010;153:256 261.

29. Swetz KM, Freeman MR, AbouEzzeddine OF et al. Palliative medicine consultation for preparedness planning in patients receiving left ventricular assist devices as destination therapy. Mayo Clin Proc 2011;86:493-500. 